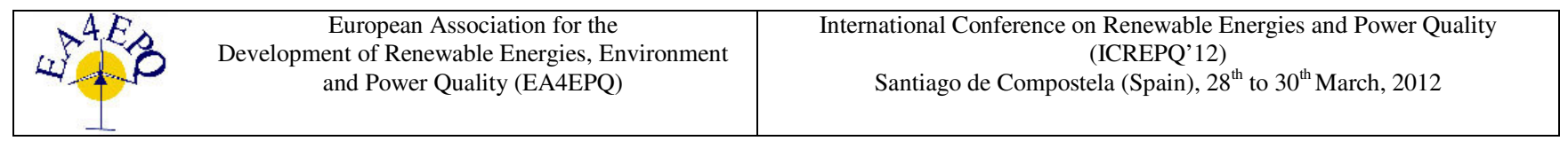

\title{
Voltage Dip Measurements along MV lines vs Primary Substations Measurements
}

\author{
F. Belloni, C. Chiappa, R. Chiumeo, L. Garbero, F. Malegori and L. Tenti \\ RSE - Ricerca sul Sistema Energetico \\ Via Rubattino 54, 20134 Milano (Italy)
}

Phone/Fax number:+003902 3992.5126, e-mail: federicoriccardo.belloni@rse-web.it, claudio.chiappa@ rse-web.it, riccardo.chiumeo@rse-web.it, luciano.garbero@ rse-web.it, fabio.malegori@ rse-web.it, liliana.tenti@ rse-web.it

\begin{abstract}
The Italian MV networks voltage quality monitoring system has been collecting power quality data since 6 years by 600 instruments, 400 of which installed on HV/MV substations MV bus-bars and statistical representative of the network, the other 200 installed at some nodes of the MV lines [1]. The paper is focused on the analysis of voltage dips monitored at some of the 200 measuring points considered more significant. The aims were to confirm, on a wider scale, the representativeness of the statistics gathered on the HV/MV stations MV bus-bars with respect to that expected along the MV lines and to investigate the events way of propagation along different voltage levels. The analysis has been performed by both statistical methods on real data and DIgSILENT ${ }^{1}$ simulations. The possibility to get the MV network voltage dip performance along the lines by measurements at HV/MV stations level has been widely confirmed. Measurements at MV lines have also confirmed the percentages of probable HV origin voltage dips, evaluated at national and macro area levels. The voltage dips duration distributions have shown a good accordance with the typical tripping times of the $\mathrm{HV}$ and MV protection system.
\end{abstract}

\section{Key words}

Power Quality, Dip Performance, Monitoring System, Protection System.

\section{Introduction}

The Italian system for Power Quality (PQ) monitoring of Medium Voltage (MV) distribution networks is thoroughly described in [1] and [2].

The PQ data monitored are referred to 400 Measurements Units (MU) installed at HV/MV primary substations, which can be considered statistically representative of the MV network (Fig.1 a), and to $200 \mathrm{MV}$ points of common coupling along the MV lines, freely chosen by MV customers and by DNOs (Distribution Network Operators) (Fig.1 b). Among these last ones, the most significant MUs are those installed along the MV lines connected to those primary substations characterized by monitored MV bus-

\footnotetext{
${ }^{1}$ DigSilent Power Factory ver. 14.0 build 523.
}

bars: the set of these MUs is usually called "grappolo", in Italian, or cluster (Fig. 2).

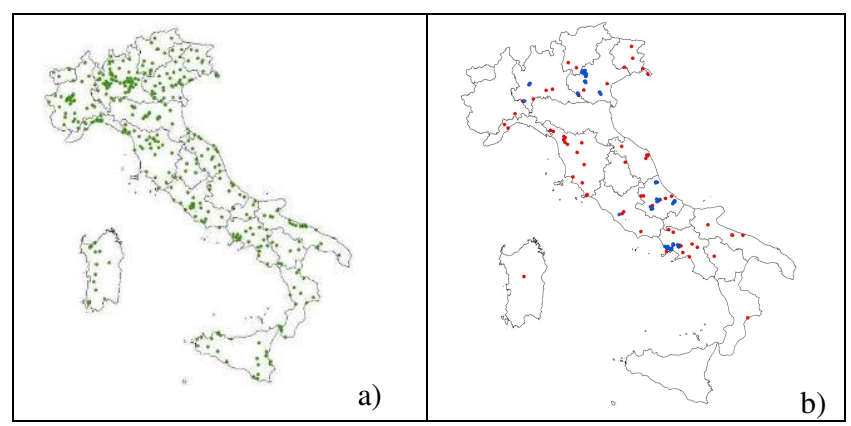

Fig. 1. (a) 400 MUs installed on the MV bus-bars (b) 200 MUs installed by the MV Customers (in red) and along the lines by the Utilities (in blue)

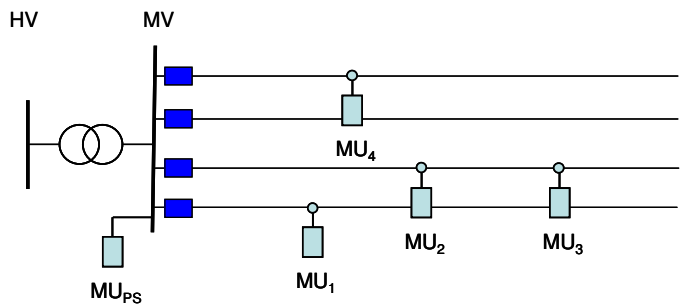

Fig. 2. "Grappolo" or cluster structure

The analysis presented hereafter mainly refers to the voltage dips monitored at some "grappoli" (clusters) with the aims to confirm, on a wider scale, the representativeness of the statistics gathered on the HV/MV stations MV bus-bars with respect to that expected along the MV lines, and to investigate the events way of propagation along different voltage levels.

\section{Measurements representativeness at primary substations level by voltage dips propagation along "grappoli"}

The first aim of the dip performance evaluation at "grappoli" level was to confirm the statement that

\footnotetext{
2 "Grappoli" in the plural.
} 
voltage dip statistics based on measurements on HV/MV primary substations MV bus-bars is a good estimation of what happens along the lines at the delivery points. This statement had been at the base of the choice of monitoring the Medium Voltage network at the primary substation level to get precise voltage quality evaluations, above all on voltage dips, by means of a reasonable number of MUs at MV level which could represent a sample of sites statistically representative of the network.

The analysis refers to the data collected during a one year period by a sample of 50 MUs belonging to different "grappoli". A similar investigation was performed in 2006, even if over a smaller number of instruments, spread on a limited geographical area, and over a shorter monitoring period.

In particular, the analysis has been focused on the assessment of both the correspondence between the number of events monitored at Primary Substation (PS) and those monitored along the lines and the voltage dips characteristics (duration and residual voltage) propagation along the lines. In this context the distribution of the ratios of the residual voltage measured on the delivery points $\left(\mathrm{U}_{\text {res_c }}\right)$ along the MV lines to that measured on the MV bus-bar $\left(\mathrm{U}_{\text {res_b }}\right)$ for the same events has been investigated (Fig. 3). As to the number of events monitored at PS level it results slightly higher than that expected along the lines due to the interruptions occurring in the lines.

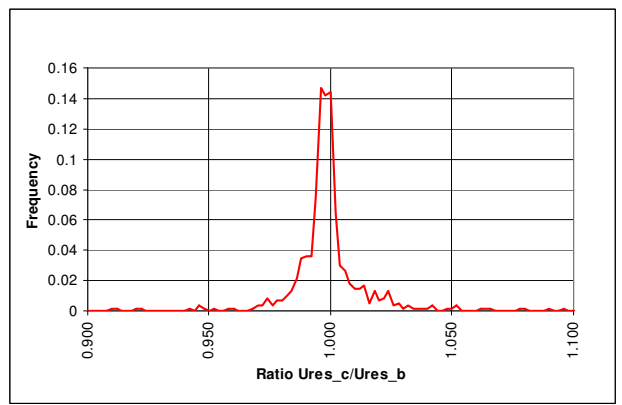

Fig. 3. Distribution of the ratio of $\mathrm{U}_{\text {res_c }}$ (delivery points) to $\mathrm{U}_{\text {res_b }}$ (MV bus-bars) for the same events.

So referring to voltage dips evaluation, the latest results, referred to a wider sample of instruments than that used in 2006, have confirmed the hypotheses at the base of the monitoring system realization, that is:

- the number of voltage dips measured on MV bus-bars of HV/MV substations gives an over-estimation of the number of dips to be expected in the points along the lines;

- the residual voltages measured on MV bus-bars of HV/MV substations are a good estimation of those expected at the delivery points, along the lines fed by that substation; $88 \%$ of the ratios $\left(\mathrm{U}_{\text {res-cl }} / \mathrm{U}_{\text {res-b }}\right)$ are within $\pm 2 \%$ (Fig. 3);

- $\quad 98 \%$ of the duration deviations are within $\pm 20 \mathrm{~ms}$ with a maximum of $30 \mathrm{~ms}$.

\section{MV dips origin}

In general voltage dips monitored in the MV network can be originated by events that occur:
- $\quad$ in the Medium Voltage network itself;

- $\quad$ in the High Voltage (HV) network;

- in the MV customer plants.

While in case of events due to faults in a MV customer plant the voltage measurement in itself is not generally enough to detect their origin and additional information from the plant, not always at disposal [3], are necessary, in the case of MV dips with probable origin in the High Voltage grid it's possible to estimate their percentage through:

- the detection of events correlated to a signal coming from High Voltage line distance protections, which is provided by the monitoring system;

- the "detailed analysis" of those MV monitored events characterized by correlated occurrence time and relevant to measurement units belonging to primary substations underlying a common HV grid.

The line to line voltage waveforms and their associated rms values for two MV dips with a probable HV origin detected by the "detailed analysis" are reported in Fig. 4 .

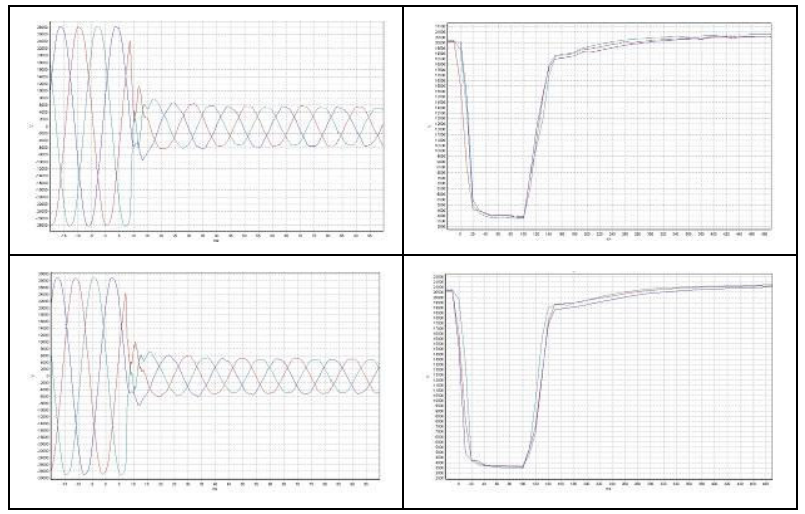

Fig. 4. Line to line rms values and waveforms of two MV dips with probable $\mathrm{HV}$ origin

This kind of analysis, performed at national level at the 400 monitored primary substations and over two years of monitoring, has led to an estimation at national level of about $27 \%$ of MV events with a probable origin in the HV grid ([4]. [5],[6] ).

By limiting the analysis to the instruments installed in the primary substations of the "grappoli", over a one year period, this percentage falls to $22 \%$. This result is not too far from the previous estimation if we take into account the fact that, in this case, the sample of measurements points can't be considered statistically representative.

Referring to the "grappoli" location restricted to 3 Italian regions, one in the north-east and the other two in the centre and south of Italy that is in 3 of the 4 macro areas defined in Fig.5 (A2=north-east; A3= Central Italy and Sardinia A4=South), the percentage of MV voltage dips of probable $\mathrm{HV}$ origin have been estimated also for those "grappoli" or clusters belonging to the same macro area as summarized in Table I . 


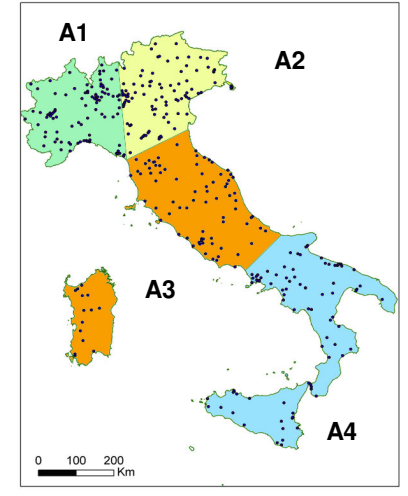

Fig. 5. Italian macro areas characterized by almost the same number of PS monitored bus-bars (A1=north-west; A2=northeast; A3= Central Italy and Sardinia A4=South)

Table I - MV voltage dip with probable origin in the HV grid

\begin{tabular}{|c|c|}
\hline $\begin{array}{c}\text { "Grappoli" } \\
\text { belonging to a } \\
\text { specific macro area }\end{array}$ & $\begin{array}{c}\text { Percentage of HV origin } \\
\text { voltage dips } \\
(\%)\end{array}$ \\
\hline Cluster_A2 & 16 \\
\hline Cluster_A3 & 28 \\
\hline Cluster_A4 & 22 \\
\hline
\end{tabular}

These percentages point out the presence of not negligible events propagation from $\mathrm{HV}$ to $\mathrm{MV}$ network also at "grappoli" level that is at the level of the instruments installed on the MV lines connected to the monitored busbars.

This fact deserves further investigations on the typical characteristics of the $\mathrm{HV}$ origin events monitored at this level. Figure 6 shows a typical scatter plot in the durationresidual voltage plane of the HV origin events as they have been monitored by the "grappoli" relevant to macro area A3 characterized by the highest percentage of these dips (28\%). The plot seems at a first sight consistent with analogous plots referred to national statistics, taking into account the reduced dimension of the statistical sample in this case ([5], [7]).

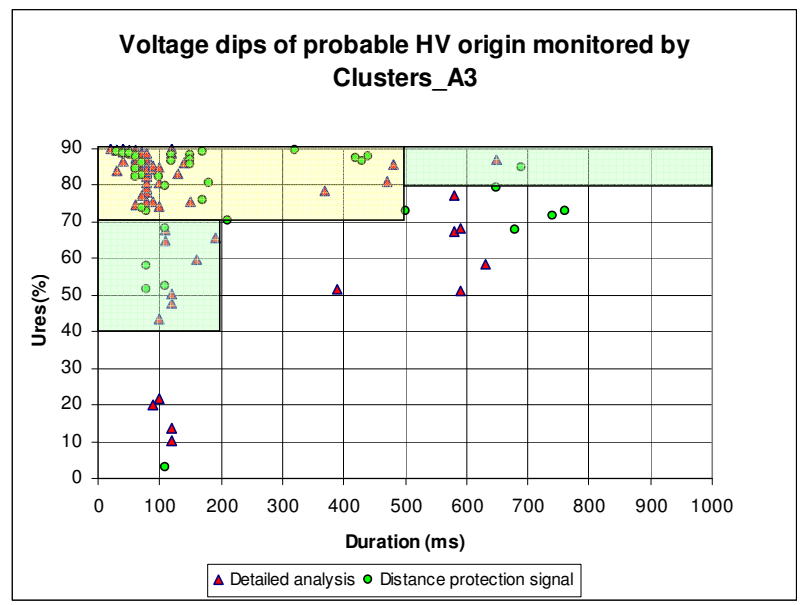

Fig. 6. Scatter plot in the duration-residual voltage plane of events of probable HV origin monitored by Cluster_A3.

Additional statistical analyses have been performed to get some number by drawing the duration distributions for these events.

\section{Duration distributions}

Referring to the measurement units of the "grappoli" belonging to the same macro area and to a one year monitoring period, events duration distributions have been calculated for voltage dips with a probable origin respectively in the MV (Fig. 7.a) and in the HV grid (Fig. 7.b) for the 3 different groups of clusters [8].

For what concern events with a probable MV origin (Fig.6 a), the distribution show peaks of probability of occurrence for duration about $120 \div 140 \mathrm{~ms}$ and $320 \mathrm{~ms}$ for all the clusters. These durations are consistent with the typical extinction time in the MV feeders protective system relevant to the $1^{\text {st }}$ zone with no intentional delay of the overcurrent protection and to its $2^{\text {nd }}$ delayed zone.

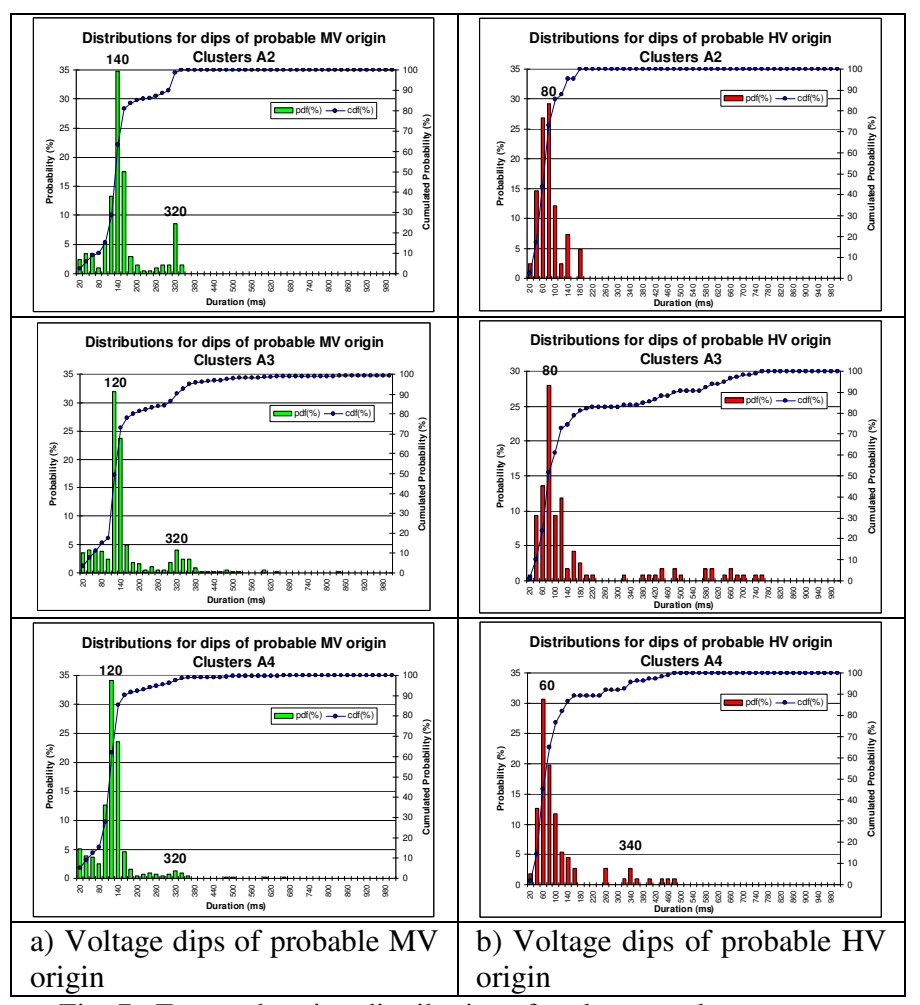

Fig. 7. Events duration distributions for clusters relevant to macro areas A2, A3, A4.

The duration distributions of events with a probable HV origin have pointed out a shorter duration peak at $60 \div 80 \mathrm{~ms}$ for all the clusters. The presence of a minor peak at about $340 \mathrm{~ms}$ has been subjected to analyses that have been performed by means of digital simulations, as described in the following paragraph.

\section{Digital simulations supporting the evaluation of voltage dips origin}

Digital simulations have been performed to support the interpretation of the results shown in the duration distributions of the MV dips with probable HV origin, paying a particular attention to the $340 \mathrm{~ms}$ peak.

In Fig. 8 a typical line to line voltage rms profile relevant to an event monitored at one of the "grappoli" primary substation which has contributed to the $340 \mathrm{~ms}$ peak is 
reported. The steady state voltage recovery is characterized by a "voltage step" delaying the achievement of the final value.

$\mathrm{V}$

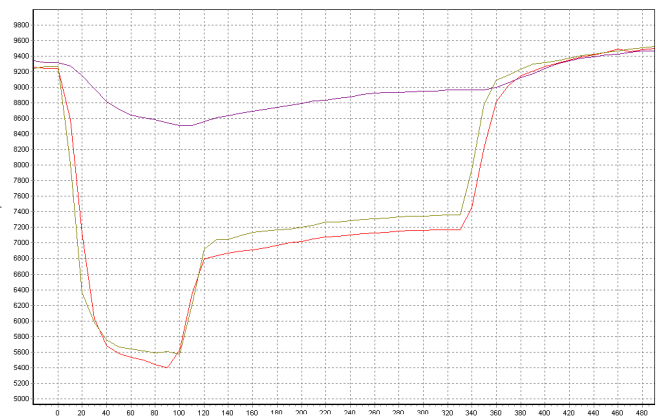

ms

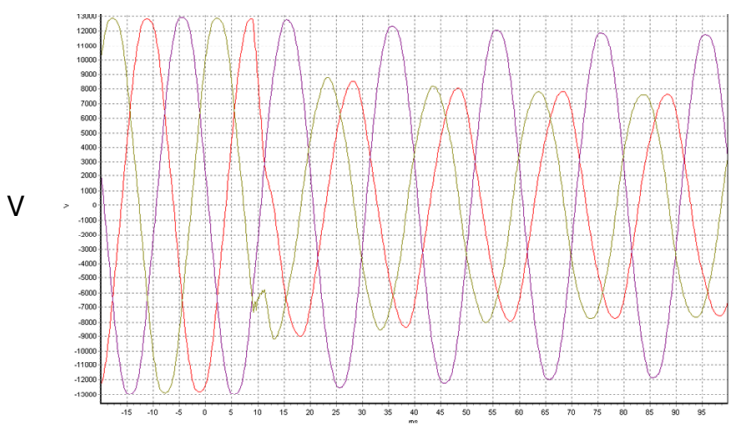

$\mathrm{ms}$

Fig. 8. Line to line voltage rms profile and initial waveform relevant to an event monitored at a primary substation.

The aim was to investigate the kind of faults in a HV grid which could be considered responsible for such a profile by making use of a HV network model developed in the DIgSILENT environment and representative of:

- a typical Italian transmission and sub-transmission network structure;

- $\quad$ generation and line protection systems;

and including a MV network and different type of faults (one phase and poly-phase faults, grounded or not).

In the different simulations performed, the rms profile of both the line to ground voltages in some AT nodes and of the line to line voltage at some MV bus bars have been analyzed on the base of some hypotheses.

Fault clearing is due to the distance relays trip at the two extreme of the faulty line, relays characterized by a tripping time depending on the measured impedance. Typically, for a HV line, this time is set at a "first step" $\left(\mathrm{t}_{1}\right)$ for fault occurred between the installation point and the $80 \%$ of the line length and a "second step" $\left(t_{2}>t_{1}\right)$ for the remaining length (Fig. 9). Moreover, it is possible to have a communication between the protection relays to extend the "first step" up to the $120 \%$ of the line length

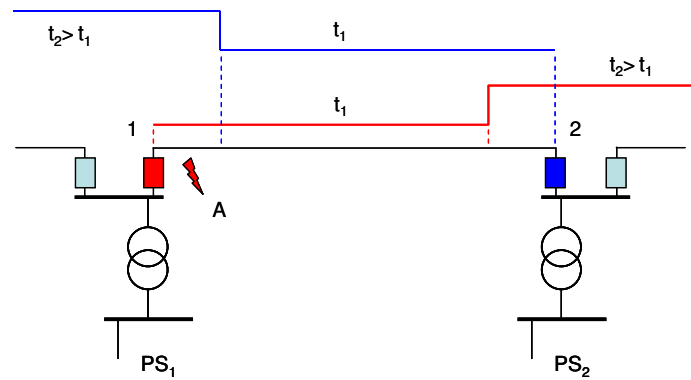

Fig. 9. Typical HV line protection scheme

Referring to the hypothesis of a possible absence of comunication, simulations have shown for one phase faults occurring in the A side of the line (Fig. 9) a protection trip in " 1 " in $\mathrm{t}_{1} \mathrm{~ms}$ and a protection trip in "2" in $\mathrm{t}_{2} \mathrm{~ms}$.

Figures 10 and 11 show respectively the rms values, associated to the event, of the:

- line voltages at the ends of the faulty line " 1 " and " 2 ";

- line to line voltages at the MV bus bars of two primary substations connected to the $\mathrm{HV}$ bar at the same points.

As primary substations belonging to "grappoli" are characterized mainly by distribution functions, according to [8], they are supposed to be provided with line distance protection systems without telecontrol, making the above-mentioned hypothesis of a lack of communication realistic.
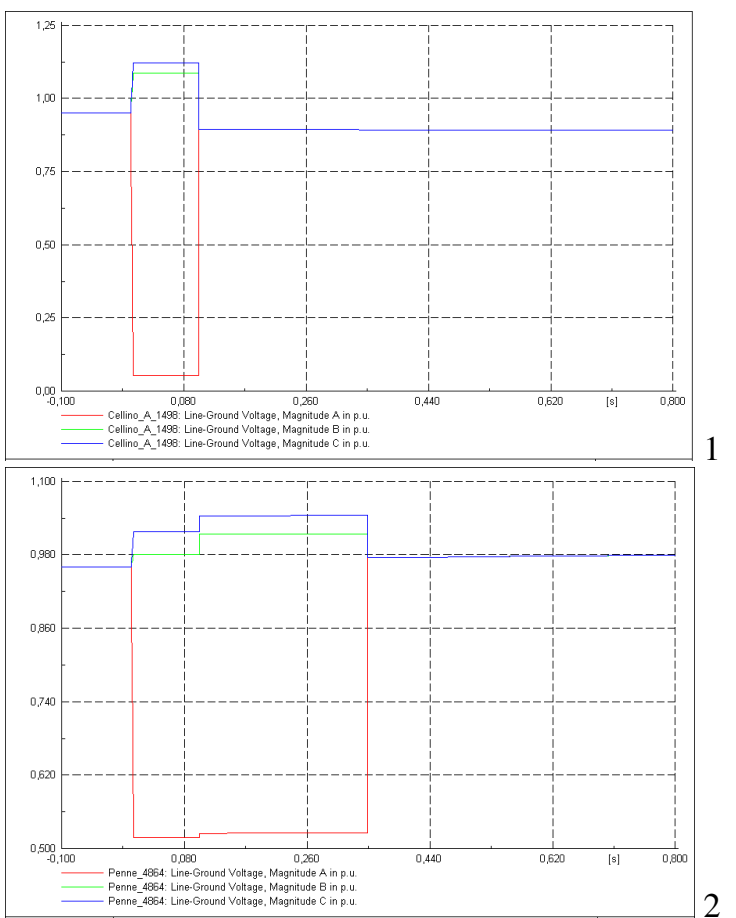

Fig. 10. Line voltages (rms) at the ends " 1 " and " 2 " of the faulty line

From the analysis of the curves, it can be pointed out that:

- $\quad$ under the above mentioned hypothesis, the simulated line to line voltages behaviour at the MV bus bar PS2 confirms the monitored data and, as a 
consequence, the possible $\mathrm{HV}$ origin of the voltage dip;

- the simulated line to line voltages at the MV bus bar PS1 show a shorter dip duration than that one of the PS2 event despite of the common HV origin;

- the simulated line voltages at the HV nodes and the relevant line to line MV voltages can be explained by the windings connection of the HV/MV transformers.

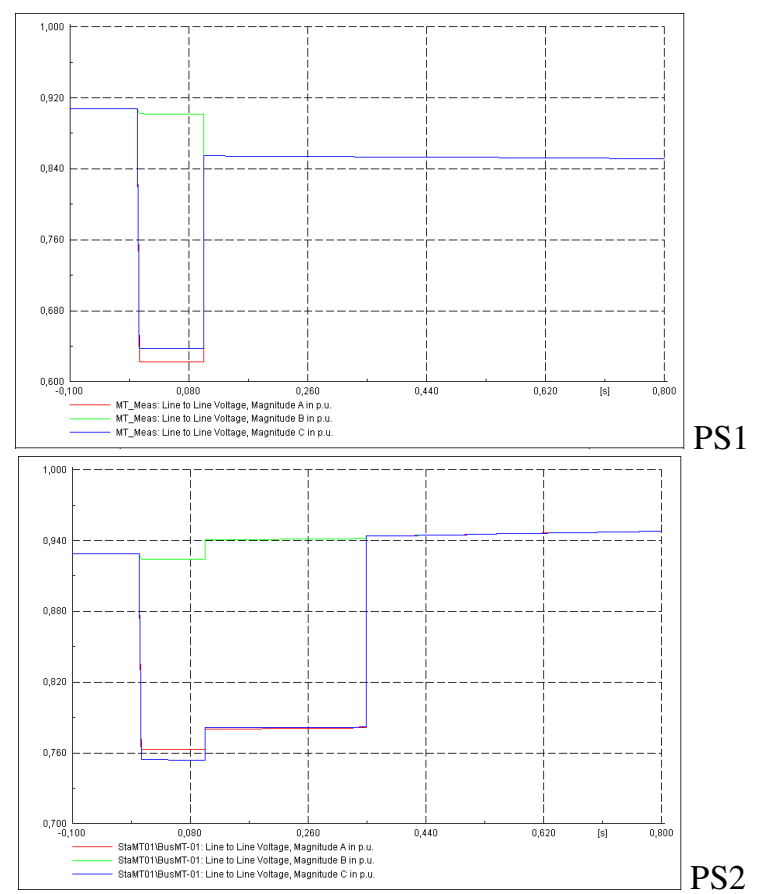

Fig. 11. Line to line voltages at the MV bus-bars of primary substation PS1 and PS2

\section{Conclusion}

The analysis of voltage dips performance monitored at "grappoli" level has confirmed, on a wider scale, the assessment on which the Italian voltage quality monitoring system has been based, that the events measurement at HV/MV substations level can be considered representative of what expected along the lines. This can be considered a very interesting result as it confirms the possibility of evaluating the MV network dip performance also along the lines by means of the MV bus-bars monitoring performed at the HV/MV substations level.

The percentages of probable HV origin voltage dips, evaluated at national and macro area level have been confirmed by the data acquired by the monitored HV/MV substations belonging to "grappoli" or clusters.

The statistical analysis of the events duration distributions together with the simulation activity performed by using DIgSILENT software show a behaviour consistent with the typical tripping times of both the $\mathrm{HV}$ and $\mathrm{MV}$ protection system.

The presence of DG along the clusters lines have not been considered in this paper but still these results can be considered particularly significant for large extension monitoring systems developed at HV/MV primary substations level.

\section{Acknowledgement}

This work has been financed by the Research Fund for the Italian Electrical System under the Contract Agreement between RSE (formerly known as ERSE) and the Ministry of Economic Development - General Directorate for Nuclear Energy, Renewable Energy and Energy Efficiency stipulated on July 29, 2009 in compliance with the Decree of March 19, 2009.

\section{References}

[1] F. Villa, A. Porrino, R. Chiumeo, S. Malgarotti, "The power quality monitoring of the MV network promoted by the Italian regulator", 19th International Conference on Electricity Distribution, CIRED 2007, Vienna, paper 0042.

[2] R. Chiumeo, L. Garbero, I. Mastandrea, O. Ornago, A. Porrino, "The Italian system for power quality monitoring of MV distribution networks" IEEE ICHQP conference 2006.

[3] Rapporto RSE 09004748 "Analisi della sensibilità dei processi industriali ai disturbi di rete", Ricerca di Sistema, Progetto "Studi sullo sviluppo del Sistema Elettrico e della Rete Elettrica Nazionale", Linea di Ricerca "Qualità della fornitura elettrica" - Febbraio 2010, www.rse-web.it, (in Italian).

[4] R. Chiumeo, A. Porrino, L. Garbero, L. Tenti, M. de Nigris, "The Italian Power Quality Monitoring System Of The MV Network: Results Of The Measurements Of Voltage Dips After 3 Years Campaign", $20^{\text {th }}$ International Conference on Electricity Distribution, CIRED 2009, Prague, paper 0737.

[5] R. Chiumeo, L. Garbero, L. Tenti, "Le prestazioni della rete in MT in relazione ai buchi di tensione", AEIT, $\mathrm{N}^{\circ} 5$, May 2011, (in Italian).

[6] Rapporto RSE 11001044 "Sistema di monitoraggio della qualità della tensione: supporto all'attività regolatoria per la definizione di una possibile rendicontazione dei buchi di tensione", Ricerca di Sistema, Progetto "Studi sullo sviluppo del Sistema Elettrico e della Rete Elettrica Nazionale", Linea di Ricerca "Qualità della fornitura elettrica" - Marzo 2011, www.rse-web.it, (in Italian).

[7] K. Brekke, M. Bollen, J. Esteves, A. Falcão, A. Haber, R. Kolessar, G. Reti, L. Lo Schiavo, F. Villa, T. Westergaard, "Monitoring of and regulations on quality of electricity supply in European countries", $20^{\text {th }}$ International Conference on Electricity Distribution, CIRED 2009, Prague, paper 0911.

[8] M. Wämundson, "Calculating voltage dips in power systems", Chalmers University of Technology, Göteborg, Sweden, 2007.

[9] GRTN (Terna) "Criteri generali di protezione delle reti a tensione uguale o superiore a $120 \mathrm{kV}$ ", Guida Tecnica $\mathrm{N}^{\circ}$ DRRPX04042 Rev. 01, 2004, (in Italian). 